\title{
Entropic Wetting and Many-Body Induced Layering in a Model Colloid-Polymer Mixture
}

\author{
Marjolein Dijkstra ${ }^{1}$ and René van Roij ${ }^{2}$ \\ ${ }^{1}$ Debye Institute, Utrecht University, Princetonpln 5, 3584 CC Utrecht, The Netherlands \\ ${ }^{2}$ Institute for Theoretical Physics, Utrecht University, Leuvenln 4, 3584 CE Utrecht, The Netherlands
}

(Received 25 June 2002; published 29 October 2002)

\begin{abstract}
We develop an efficient simulation scheme to study a model suspension of equally sized colloidal hard spheres and nonadsorbing ideal polymer coils, both in bulk and adsorbed against a planar hard wall. The many-body character of the polymer-mediated effective interactions between the colloids yields a bulk phase diagram and adsorption phenomena that differ substantially from those found for pairwise simple fluids; e.g., we find an anomalously large bulk liquid regime and, far from the bulk triple point, three layering transitions in the partial wetting regime prior to a transition to complete wetting by colloidal liquid.
\end{abstract}

DOI: $10.1103 /$ PhysRevLett.89.208303

Mixtures of nonadsorbing polymer coils and sterically stabilized colloidal spheres in a suspending medium are not only industrially important but also of profound fundamental interest. The reason is that such mixtures can be regarded as one-component systems of colloids with polymer-induced effective interactions whose strength and range can be tuned independently by varying the polymer fugacity $z_{p}$ and the size ratio $q=R_{p} / R_{c}$, respectively. Here $R_{p}$ is the polymer radius of gyration and ${ }_{R c}$ the colloidal hard-core radius. For $q \geqslant 0.25$, the bulk phase diagram of a colloid-polymer mixture exhibits, at sufficiently high $z_{p}$, a two-phase region where a colloid-dilute ("gas") and a colloid-dense ("liquid") phase coexist [1-4]. This is similar to the gas-liquid coexistence in simple fluids with $z_{p}$ playing the role of inverse temperature. However, there are profound differences from simple fluids. For instance, the cohesion that stabilizes the colloidal liquid phase is not provided by dispersion forces, but by depletion forces generated by the gain of free volume (entropy) for the polymers upon colloidal crowding [5,6]. Another difference from simple fluids is that the pairwise additivity of the effective interactions breaks down qualitatively in this $q$ regime, i.e., three-body and more-body interactions are no longer small perturbations to the pairwise interactions. A recent density functional study predicts that a model colloidpolymer mixture in this many-body regime features fascinating hard-wall adsorption phenomena that differ dramatically from those of simple liquids [7], e.g., the system exhibits several distinct layering transitions upon approach of gas saturation far from the gas-liquid-crystal triple point while the hard wall-gas interface remains partially wet. Simulation studies of these surface phenomena are nonexistent as they require the effective many-body interactions explicitly-most studies thus far involve the approximation of pairwise additivity $[4,8]$. In this Letter, we develop a novel Monte Carlo (MC) scheme for a model colloid-polymer mixture, based on the exact effective colloid Hamiltonian; i.e., it incorporates all many-body interactions. Employing this
PACS numbers: 82.70.Dd, 64.60.-i, 68.08.-p

scheme, we are able to study not only the full phase diagram and the structure of the bulk, but also wetting and layering phenomena near a hard wall for arbitrary values of $q$. Results are presented for $q=1$.

The essential ingredient of colloid-polymer interactions is the depletion of polymers in a shell of order $R_{p}$ about an impenetrable colloidal surface, caused by the reduction of the number of polymer configurations (i.e., the entropy) in the shell. Asakura and Oosawa (AO) realized that this depletion effect should lead to an effective attraction between two colloids if their depletion layers overlap, i.e., if their surface-surface distance is smaller than about $2 R_{p}$ [5]. The simplest model that catches the essence of polymer-induced depletion interactions between colloidal hard spheres is due to Vrij [6] (although it is called the AO model). Vrij described the polymers as noninteracting interpenetrating spheres as regards their mutual interactions, while the colloidpolymer pair interaction is hard-sphere-like such that their distance of closest approach is $R_{c p} \equiv R_{c}+R_{p}$. In this Letter, we study this simple model in yet arbitrary external potentials $U_{c}(\mathbf{r})$ and $U_{p}(\mathbf{r})$ for the colloids and the polymers, respectively. It proves most convenient to treat the polymers grand canonically, at fixed fugacity $z_{p}$. This ensemble is such that the statistical weight $\exp [-\beta H]$ of $N_{c}$ colloids, with coordinates $\mathbf{R}_{i}, i=1$, $\ldots, N_{c}$, is given in terms of the effective Hamiltonian $H=\sum_{i<j}^{N_{c}} u_{c}\left(R_{i j}\right)+\sum_{i=1}^{N_{c}} U_{c}\left(\mathbf{R}_{i}\right)+\Omega\left(\left\{\mathbf{R}_{i}\right\} ; z_{p}, \beta\right)[4,9]$. Here $\beta$ is the inverse temperature, $u_{c}$ the colloid-colloid hard-sphere potential, $R_{i j}=\left|\mathbf{R}_{i}-\mathbf{R}_{j}\right|$, and $\Omega$ the grand potential of the polymers in the external potential $U_{p}$ and in the static configuration $\left\{\mathbf{R}_{i}\right\}$ of the colloidal spheres. Because of the ideal character of the polymer-polymer interactions, one obtains for the present model the exact result $\beta \Omega=-z_{p} V_{f}$, with the so-called free volume

$$
V_{f}=\int d \mathbf{r} \exp \left[-\beta U_{p}(\mathbf{r})\right] \prod_{i=1}^{N_{c}}\left[1+f\left(\left|\mathbf{r}-\mathbf{R}_{i}\right|\right)\right] .
$$

Here the integral is over the total volume $V$ of the 
suspension, and $f(r)$ is the colloid-polymer Mayer function, which for the present model equals -1 for $0<r<$ $R_{c p}$, and 0 otherwise. Nonvanishing contributions to $V_{f}$ stem from those positions $\mathbf{r}$ that are outside any of the $N_{c}$ depletion shells. The shape of the free volume is, in general, highly irregular and nonconnected, and, hence, an accurate evaluation of $V_{f}$, e.g., by a finite element method, is computationally extremely demanding. In order to increase the numerical accuracy and computational speed, we expand the product in Eq. (1), and separate the $\mathcal{O}\left(f^{k}\right)$ terms with $k=0,1,2$ (for which analytic expressions are possible for some $U_{p}$ ) from those with $k \geq 3$,

$$
V_{f}=V^{(0)}+\sum_{i=1}^{N_{c}} V^{(1)}\left(\mathbf{R}_{i}\right)+\sum_{i<j}^{N_{c}} V^{(2)}\left(\mathbf{R}_{i}, \mathbf{R}_{j}\right)+V_{f}^{(3+)},
$$

with the accumulation of the $k \geq 3$ terms,

$$
V_{f}^{(3+)}=\sum_{k \geq 3}\left[\sum_{i_{1}<\cdots<i_{k}}^{N_{c}} V^{(k)}\left(\mathbf{R}_{i_{1}}, \ldots, \mathbf{R}_{i_{k}}\right)\right] .
$$

Here $V^{(0)}=\int d \mathbf{r} \exp \left[-\beta U_{p}(\mathbf{r})\right]$, and, for $k \geq 1$,

$$
V^{(k)}=\int d \mathbf{r} \exp \left[-\beta U_{p}(\mathbf{r})\right] \prod_{m=1}^{k} f\left(\left|\mathbf{r}-\mathbf{R}_{i_{m}}\right|\right) .
$$

Equation (2) is the decomposition of $V_{f}$, and, hence, of the polymer-induced colloidal interactions $\Omega$, into $k$-body contributions. In a translational invariant bulk system, where $U_{p} \equiv 0$, the $k \leq 2$ terms of Eq. (2) are easily obtained analytically, with $V^{(0)}=V, V^{(1)}=-v_{1}$, with $v_{1}=(4 \pi / 3) R_{c p}^{3}$ the volume excluded to a polymer by a single colloid, and $V^{(2)}\left(\mathbf{R}_{i}, \mathbf{R}_{j}\right)=v_{1}\left(1-3 x / 4+x^{3} / 16\right)$, with $x=R_{i j} / R_{c p}<2$, the lens-shaped overlap volume of two spheres of radius $R_{c p}$ at separation $R_{i j}$. Note that these $k=0,1$ terms are irrelevant offsets that do not affect the thermodynamics and structure of the bulk suspension [9], and that $-z_{p} \beta^{-1} V^{(2)}\left(R_{i j}\right) \equiv u_{d}\left(R_{i j}\right)$ is the well-known depletion potential of the AO model $[1,6]$. The pairwise additivity approximation is recovered when $V_{f}^{(3+)}$ is set to zero. Here we go beyond this approximation by a numerical evaluation of $V_{f}^{(3+)}$; the $k \leq 2$ terms are treated analytically. For the present model, an efficient scheme can be constructed by introducing $n=n(\mathbf{r}) \equiv-\sum_{i=1}^{N_{c}} f\left(\left|\mathbf{r}-\mathbf{R}_{i}\right|\right)$, the number of simultaneously overlapping depletion layers in $\mathbf{r}$. Noting that the product of the $k$ Mayer functions in Eq. (4) is only nonvanishing [and equals $(-1)^{k}$ ] for $n ! / k !(n-k)$ ! terms of the summation over $i_{1}, \ldots, i_{k}$ in Eq. (3), one performs the $k$ summation of Eq. (4) to obtain

$$
V_{f}^{(3+)}=-\frac{1}{2} \int_{n \geq 3} d \mathbf{r} \exp \left[-\beta U_{p}(\mathbf{r})\right][n(\mathbf{r})-1][n(\mathbf{r})-2],
$$

where the integration is only over those regions where $n(\mathbf{r}) \geq 3$. Note that Eq. (5) holds strictly for the present Mayer function, whereas Eqs. (1)-(4) hold for any $f$ provided the polymer-polymer interaction is ideal. One could, now, envisage a numerical calculation of $V_{f}^{(3+)}$, and, hence, of $H$, by a finite element method, whereby the total volume $V$ is covered by $M$ cells centered at grid points $\mathbf{r}_{m}$ for $m=1, \ldots, M$. The computational speed can be greatly improved if one realizes that the standard Metropolis algorithm for MC simulation is based on an acceptance probability $\min (1, \exp [-\beta \Delta H])$, with $\Delta H$ the change of $H$ due to a proposed configuration change. This implies that only the corresponding change $\Delta V_{f}^{(3+)}$ is required, and not $V_{f}^{(3+)}$ itself. For MC moves involving only a single colloid, for example $\mathbf{R}_{1} \rightarrow \mathbf{R}_{1}^{\prime}$, the only contributions to $\Delta V_{f}$, and, hence, to $\Delta V_{f}^{(3+)}$, occur inside the two spheres of radius $R_{c p}$ centered about $\mathbf{R}_{1}$ and $\mathbf{R}_{1}^{\prime}$. We mesh these two spheres by typically $M \simeq 2.5 \times 10^{5}$ gridpoints each, which we considered the optimal balance between accuracy and computational speed. We then calculate $n\left(\mathbf{r}_{m}\right)$ for all $\mathbf{r}_{m}$, and estimate $\Delta V_{f}^{(3+)}$ using Eq. (5). If $V^{(k)}$ cannot be determined analytically for $k=2,1$ because of a nontrivial $U_{p}(\mathbf{r})$, one can derive expressions similar to Eq. (5) for $V_{f}^{(2+)}$ or $V_{f}^{(1+)}$.

On the basis of our MC scheme, we first performed bulk free energy calculations of the $q=1$ AO model using thermodynamic integration at a fixed colloid packing fraction $\eta=(4 \pi / 3) R_{c}^{3} N_{c} / V$, such that the polymer fugacity is switched on from 0 to the $z_{p}$ of interest [9]. The resulting bulk phase diagram, constructed from common tangent constructions at fixed dimensionless fugacity $\phi=(4 \pi / 3) R_{p}^{3} z_{p}$, exhibits gas, liquid, and crystalline phases, with a gas-liquid critical point at $\phi_{c} \simeq 0.70$ and a gas-liquid-crystal triple point at $\phi_{t} \simeq 6.0$. Note that $\phi$ is the polymer packing fraction in the reservoir at fugacity $z_{p}$, and that the ratio $\phi_{t} / \phi_{c} \simeq 9$ is remarkably high compared to the corresponding (inverse) temperature ratio in simple fluids. Figure 1 shows the phase boundaries in the $\eta-\phi$ representation. The full curve denotes that part of the saturated gas branch that is relevant for our wetting and layering study (to which the symbols and the line thickness refer), and the symbols ( $\square$ ) of the inset denote gas-liquid and fluid-solid binodals at full scale. The dashed curves are the phase boundaries as predicted by the "free volume theory" of Ref. [10], where $V_{f}$ is accounted for within first-order perturbation theory by its average in a pure hard-sphere system. At full scale, see inset of Fig. 1, this approximation turns out to be remarkably accurate. However, on the expanded scale of the gas branch, the deviations are appreciable, e.g., a factor of about 2 in $\eta$ at $\phi=0.9$. Comparing the present "exact" phase diagram for $q=1$ with the one based solely on pairwise AO interactions, for which the triple and critical point values of $\phi$ are $\sim 0.8$ and $\sim 0.5$, respectively [4], shows that the main effect of the many-body interactions is to enhance the $\phi$ regime of stable gas-liquid coexistence considerably at the expense of that of the gas-solid coexistence. Finally, we remark that, although we did not simulate the polymers explicitly, we obtained the three 


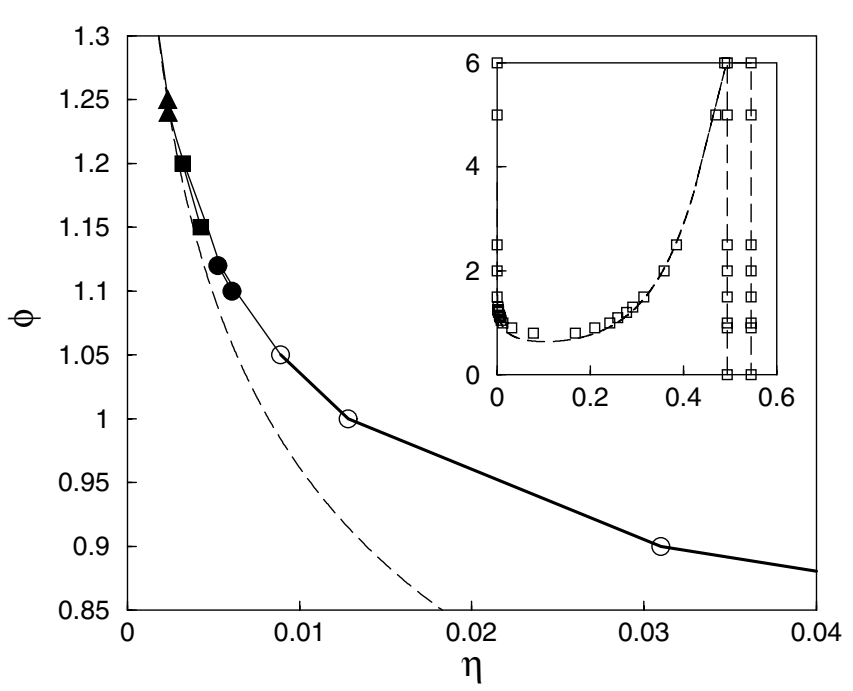

FIG. 1. Bulk and surface phase diagram of the AO model (size ratio $q=1$ ) as a function of the colloid packing fraction $\eta$ and the polymer reservoir packing fraction $\phi$. The main figure is a blowup of the saturated bulk gas branch, separated into a regime of complete wetting (thick curve, $\circ$ ), and partial wetting by colloidal liquid (thin curve) at a hard planar wall. The first $(\boldsymbol{\Delta})$, second $(\boldsymbol{\square})$, and third $(\bullet)$ layering transitions are off bulk coexistence. The inset shows the gas-liquid and fluid-solid bulk coexistence ( $\square$ ) of this work to full scale. The dashed curves denote the bulk binodals of the "free volume theory" [10].

partial structure factors and radial distributions from measuring colloid-colloid, colloid-hole, and hole-hole correlations (not shown here).

Next, we consider the AO model with $q=1$ in contact with a planar hard wall at $z=0$, i.e., such that $U_{p}(z)=$ $U_{c}(z)=0$ for $z>R_{c}\left(=R_{p}\right)$ and $\infty$ for $z<R_{c}$, with $z$ the distance of a particle from the wall. One then finds from Eq. (4) for $k=1$ that the effective, polymer-induced wall-colloid potential $-z_{p} \beta^{-1} V^{(1)}(z)$ is attractive if $z<$ $R_{p}+R_{c p}$, with a strength similar to that of the bulk colloid-colloid depletion potential $u_{d}\left(R_{i j}\right)$ [8]. On the basis of the attractive one-body potential, one expects preferential colloid adsorption by the hard wall. Moreover, given the large $\phi$ interval $\left[\phi_{c}, \phi_{t}\right]$ with a stable gasliquid binodal, one might expect, by analogy with simple fluids, a wetting transition at some $\phi=\phi_{w}$ in this interval [11]. However, unlike the case of simple fluids, the pair interaction $-z_{p} \beta^{-1} V^{(2)}\left(\mathbf{R}_{i}, \mathbf{R}_{j}\right)$ is now a nontrivial function of $\mathbf{R}_{i}$ and $\mathbf{R}_{j}$ for two colloids close to the wall, with a strength that is reduced considerably compared to its translationally invariant bulk form $u_{d}\left(R_{i j}\right)$ [8]. This reduction, which is caused by overlap of the pairwise "lens" with the wall, tempers the tendency to form a dense liquid layer at contact, and thus competes with the attractive one-body potential. The effect of the $k$-body interactions with $k \geq 3$ on the adsorption is less clear. Here we investigate the adsorption with a grand canonical version of our MC scheme, which includes all many-body effects. We measured the equilibrium colloid density profile $\eta(z)$ at fixed $\phi$ in a rectangular box of volume $A \times L$. Here $A$ is the area of the hard wall, along which we apply periodic boundary conditions, and $L=100 R_{c}$ the box length perpendicular to the wall such that $0<z<L$. We use the technique of Ref. [12] to impose a flat average profile $\eta(z)=\eta$ for $L-4 R_{c} \leq$ $z \leq L$, where the bulk packing fraction $\eta$ is fixed by the chemical potential. For all state points considered, the density profile is found to be constant in $20 R_{c}<z<L$, i.e., we mimic a semi-infinite bulk gas in contact with a hard wall.

In Fig. 2, we show the profiles $\eta(z)$ in the vicinity of the hard wall, for $\phi=0.90$ [2(a)] and $\phi=1.12$ [2(b)], at several $\eta<\eta_{\text {sat }}$, the saturated gas density. The insets show the corresponding dimensionless adsorption $\Gamma=\int_{0}^{L} d z[\eta(z)-\eta] / R_{c}$. The formation of a thick liquid film and the logarithmic increase of $\Gamma$ as $\eta \rightarrow \eta_{\text {sat }}$ in Fig. 2(a) are strongly indicative of complete wetting at $\phi=0.90$. From the slope of the inset in 2(a), one obtains the correlation length $\xi=(1.16 \pm 0.02) R_{c}$ of the wetting phase [11], which agrees well with $\xi=(1.20 \pm 0.08) R_{c}$ as determined from the asymptotic decay of the radial distribution function of the bulk coexisting liquid at the value of $\phi$. Evidence for complete wetting was also found for $\phi=1.00$ and 1.05. By contrast, the finite $\Gamma$ for $\eta \rightarrow \eta_{\text {sat }}$ and the finite liquid film thickness in Fig. 2(b) imply partial wetting at $\phi=1$.12. Indeed partial wetting was observed for all $\phi \geq 1.10$ that we considered. This implies that the wetting transition occurs at $\phi_{w}$ with $1.05<\phi_{w}<1.10$. Despite considerable effort, we did not find any evidence for a prewetting transition, i.e., a transition from a thin to a thick liquid film, which by analogy with simple liquids is to be expected in the complete wetting regime $\phi \leq \phi_{w}$ if the wetting transition is first order [11]. Surprisingly, however, we did find off-coexistence jumps $\delta \Gamma$ in $\Gamma$ in three separate regimes $\phi>\phi_{w}$, i.e., in the partial wetting regime. The inset of Fig. 2(b) shows such a jump, which we associate, following Ref. [7], with a layering transition (even though the adsorption is not strictly localized in a well-defined layer). From the profiles in Fig. 2(b), one checks that $\delta \Gamma$ is due to a condensation in a regime $4 R_{c} \leq z \leq$ $12 R_{c}$. This "third" layering transition, indicated by $\bullet$ in Fig. 1, was also found at $\phi=1.10$, although slightly further off coexistence and with a smaller $\delta \Gamma$. At yet smaller $\phi$, we did not detect any discontinuity in the adsorption, consistent with a low- $\phi$ critical point for a line of layering transitions. We found similar adsorption discontinuities associated with the second $(\phi=1.15$, $1.20)$ and first $(\phi=1.24,1.25)$ layering transition, as indicated by $\boldsymbol{\square}$ and $\boldsymbol{\Delta}$, respectively. The jump in the first is due to adsorption in $1.5 R_{c} \leq z \leq 6 R_{c}$, and that in the second in $3 R_{c} \leq z \leq 8 R_{c}$. Note that all three layering transitions take place just below saturation, are entropy driven, occur only for $\phi_{w}<\phi \ll \phi_{t}$, extend over a rather small regime of $\phi$, and seem to end in their own (surface) 

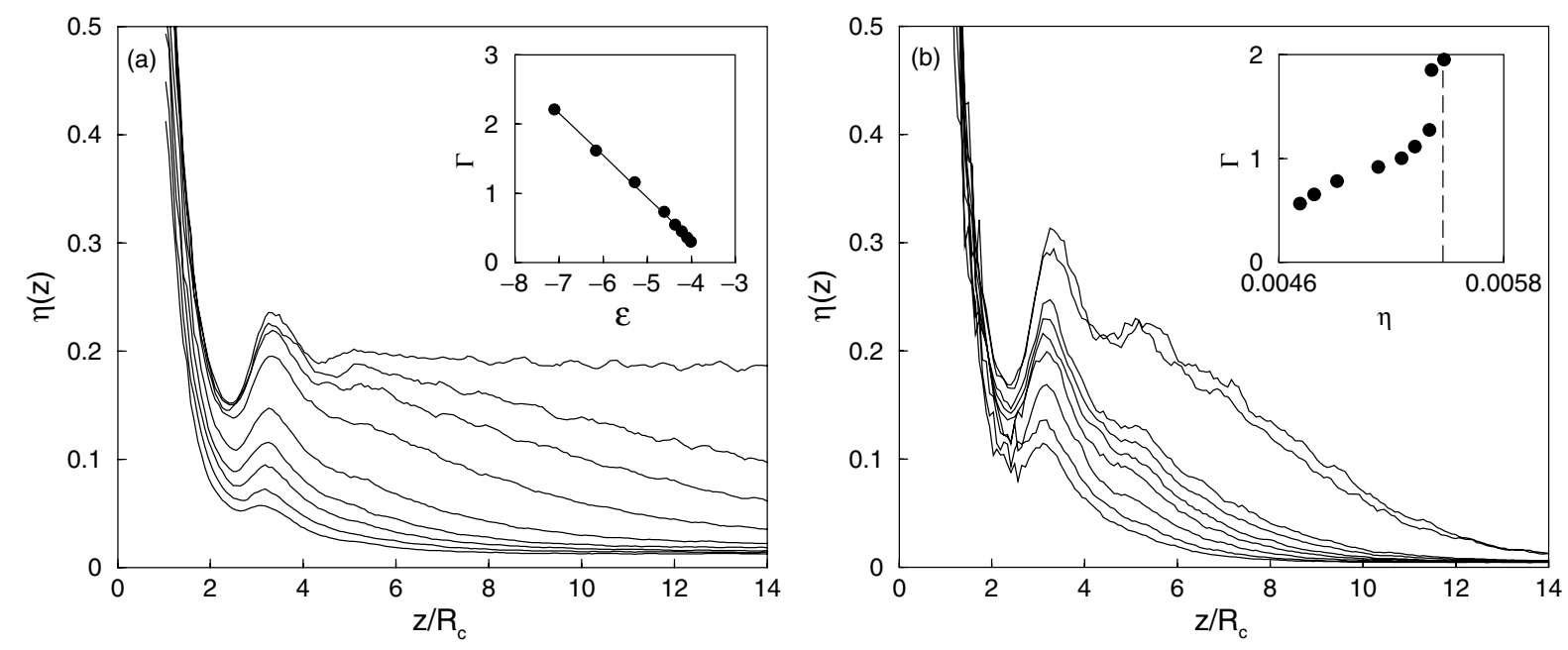

FIG. 2. Colloid density profiles $\eta(z)$, for several fugacities (asymptotic bulk densities $\eta$ ), near a hard wall at $z=0$. The reservoir polymer packing fraction is $\phi=0.90$ (a), and $\phi=1.12$ (b). Upon approach of gas saturation, the adsorption $\Gamma$ appears to diverge as $-\epsilon$, with $\epsilon \equiv \ln \left(\eta_{\text {sat }}-\eta\right)$ in (a), i.e., complete wetting. $\Gamma$ remains finite at $\eta_{\text {sat }}$ (dashed vertical line) in (b), i.e., partial wetting. The jump of $\Gamma$ prior to saturation is associated with the distinct change in the density profiles and reflects the third layering transition.

critical point. Currently, we have no detailed understanding of the mechanism behind these layering transitions, which take place in the partial wetting regime far from the triple point, and not, as in simple fluids at strongly adsorbing substrates, in the complete wetting regime close to the triple point [13]. It is tempting to speculate, however, that the competition between the one- and twobody interaction (and perhaps that between two- and three-body terms, etc.) should be important. Since our results are in qualitative agreement with recent density functional predictions for the AO model [7], this suggests that the wetting and layering are robust phenomena in this model of colloid-polymer mixtures. Hopefully, this stimulates experimental investigations.

A few final words on the system sizes used in this work. In all cases we fixed $L=100 R_{c}$, and varied the area $A$ such that the typical relative adsorption jumps $\delta \Gamma / \Gamma \simeq 0.3$ can be resolved numerically with confidence. This implies that $\delta \eta / \eta$, with $\delta \eta$ the statistical error in $\eta$ at the jump, is substantially smaller than 0.3 ; it turns out that $\delta \eta / \eta=0.05$ is (just) sufficient. Consequently, one requires on average at least 400 colloids in the grand canonical bulk. Given that $\eta \lesssim 5.10^{-3}$ here, one requires $A \gtrsim(60 \times 60) R_{c}^{2}$. Hence, the total number of colloids in the system exceeds $10^{3}$, since $\Gamma \simeq 1$. If one had wished to study wetting and layering of the two-component colloidpolymer mixture directly (instead of using the present effective one-component colloid description), one would have needed $10^{5}$ polymers (most of them filling the bulk). This shows that our approach, based on effective (manybody) interactions, is much more efficient.

In conclusion, we have shown with an efficient (and extendable) numerical scheme that entropic many-body effects change dramatically the adsorption phenomena for colloid-polymer mixtures from those for simple (pairwise) fluids.

It is a pleasure to thank R. Evans and J. Brader for stimulating discussions. This work is part of the Research program of the "Stichting voor Fundamenteel Onderzoek der Materie (FOM)," which is financially supported by the "Nederlandse Organisatie voor Wetenschappelijk Onderzoek (NWO)."

[1] A. P. Gast, C. K. Hall, and W. B. Russel, J. Colloid Interface Sci. 96, 251 (1983).

[2] S. M. Ilett et al., Phys. Rev. E 51, 1344 (1995).

[3] E. J. Meijer and D. Frenkel, Phys. Rev. Lett. 67, 1110 (1991); J. Chem. Phys. 100, 6873 (1994).

[4] M. Dijkstra, J. M. Brader, and R. Evans, J. Phys. Condens. Matter 11, 10079 (1999).

[5] S. Asakura and F. Oosawa, J. Chem. Phys. 22, 1255 (1954).

[6] A. Vrij, Pure Appl. Chem. 48, 471 (1976).

[7] J. M. Brader et al., J. Phys. Condens. Matter 14, L1 (2002); J. M. Brader, Ph.D. thesis, University of Bristol, 2001.

[8] J. M. Brader, M. Dijkstra, and R. Evans, Phys. Rev. E 63, 041405 (2001).

[9] M. Dijkstra, R. van Roij, and R. Evans, Phys. Rev. E 59, 5744 (1999).

[10] H. N.W. Lekkerkerker et al., Europhys. Lett. 20, 559 (1992).

[11] M. Schick, in Liquids at Interfaces, edited by J. Charvolin et al. (Elsevier, Amsterdam, 1990).

[12] M. Dijkstra, R. van Roij, and R. Evans, Phys. Rev. E 63, 051703 (2001).

[13] P. C. Ball and R. Evans, J. Chem. Phys. 89, 4412 (1988). 